\title{
3D automatic quantification applied to optically sectioned images to improve microscopy analysis
}

\author{
J.E. Diaz-Zamboni, J.F. Adur, N. Vicente, M.P. Fiorucci, M.F. Izaguirre, V.H. Casco \\ Laboratorio de Microscopía, Facultad de Ingeniería, Universidad Nacional de Entre Ríos, Oro Verde, Entre \\ Ríos, Argentina
}

il

(C)2008 European Journal of Histochemistry

New fluorescence microscopy techniques, such as confocal or digital deconvolution microscopy, allow to easily obtain three-dimensional (3D) information from specimens. However, there are few 3D quantification tools that allow extracting information of these volumes. Therefore, the amount of information acquired by these techniques is difficult to manipulate and analyze manually.

The present study describes a model-based method, which for the first time shows 3D visualization and quantification of fluorescent apoptotic body signals, from optical serial sections of porcine hepatocyte spheroids correlating them to their morphological structures.

The method consists on an algorithm that counts apoptotic bodies in a spheroid structure and extracts information from them, such as their centroids in cartesian and radial coordinates, relative to the spheroid centre, and their integrated intensity. 3D visualization of the extracted information, allowed us to quantify the distribution of apoptotic bodies in three different zones of the spheroid.

Key words: fluorescence, deconvolution, 3D quantification, information extraction.

Correspondence: Victor H. Casco,

Laboratorio de Microscopía. Facultad de Ingeniería.

Universidad Nacional de Entre Ríos, Oro Verde,

Entre Ríos, Argentina

E-mail: vcasco@bioingenieria.edu.ar

Paper accepted on April 4, 2008

European Journal of Histochemistry

2008; vol. 52 issue 2 (Apr-Jun): 115-126
T hree-dimensional (3D) analyses of biological specimens have represented one of the major achievements of modern biology. It is a crucial prerequisite for understanding complex biological assemblies (Agard, 1984). This, in association with the development of tools for precise quantification (Arndt-Jovin, 1985), would seem to be completely known. However, typical volume analyses are still done, using physical-sectioning techniques combined with stereological methods. Those techniques naturally produce loss of 3D information, which in turn, affect the details of microscopy images. In addition, the human brain is not particularly good reconstructing $3 \mathrm{D}$ structural information from a stack of bi-dimensional (2D) slices (Chen, 1995). Hence, the 2D analysis of individual sections is not a good approach to obtain information of the 3D structural functional data. In addition, this is worsened when 3D quantitative analyses are required.

3D analyses difficulties have been partially solved by new fluorescence microscopy techniques, such as confocal or digital deconvolution microscopy, which have allowed to easily obtain 3D information from specimens. Currently, the amount of 3D information obtained with these techniques is greater than that obtained with 2D conventional techniques, but this has begun to be a problem, since it is very difficult to manipulate and analyze it.

Initially, we implemented digital deconvolution microscopy techniques to expand our studies from the $2 \mathrm{D}$ to the $3 \mathrm{D}$ domain. These combine computerbased methods with optical sectioning (Adur and Schlegel, 1997) and make 3D visualization of fluorescent biological signals possible. The system is controlled by a specialized software, SUMDD (from the Spanish: Software para Usuarios de Microscopía de Desconvolución Digital) (DiazZamboni, 2004). A typical session of the system consists on the following steps: optical sectioning of the specimen, point spread function (PSF) determination, deconvolution, 3D visualization and quantifi- 
cation (Figure 1).

$3 \mathrm{D}$ visualization is the first step required for correct qualitative analyses. 3D quantification is a more useful tool for analyses, because it produces results that are readily comparable, enabling more precise knowledge about error propagation and, hence, much more correct statistical analyses. Finally, automatic 3D quantification raises the speed of analyses and avoids the introduction of non-systematic errors, such as fortuitous human errors. Figure 1 summarizes the structure of the system and steps followed in order to perform analyses.

In order to add an automatic 3D quantification tool that our system was lacking, we developed one based on the mathematical background of the model-based methods. In these, the model repre- sents the expected shape and local gray level structure of a target object in an image (Cootes, 2000). Specifically, a biological phenomenon is analyzed and described in a mathematical form. Once a model has been tested, it may be used to quantify as many times as necessary, for instance, for the same biological phenomena under different conditions.

We first developed a generic 3D quantification algorithm. It searches for a type of specific morphological element inside a volume. The algorithm was implemented in calculus software and it depends on a set of parameters, which must be established before processing so that it will be in accordance with the biological phenomenon under study. In the current paper, we will discuss how the model was set up, the algorithm's logic and its performance with a known biological phenomenon. For

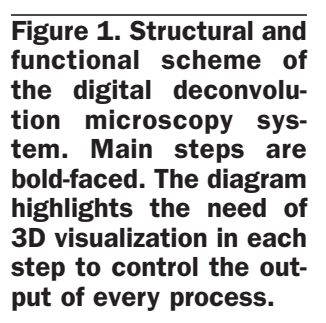

Figure 1. Structural and functional scheme of the digital deconvolumicroscopy sysThe diagram highlights the need of put of every process.

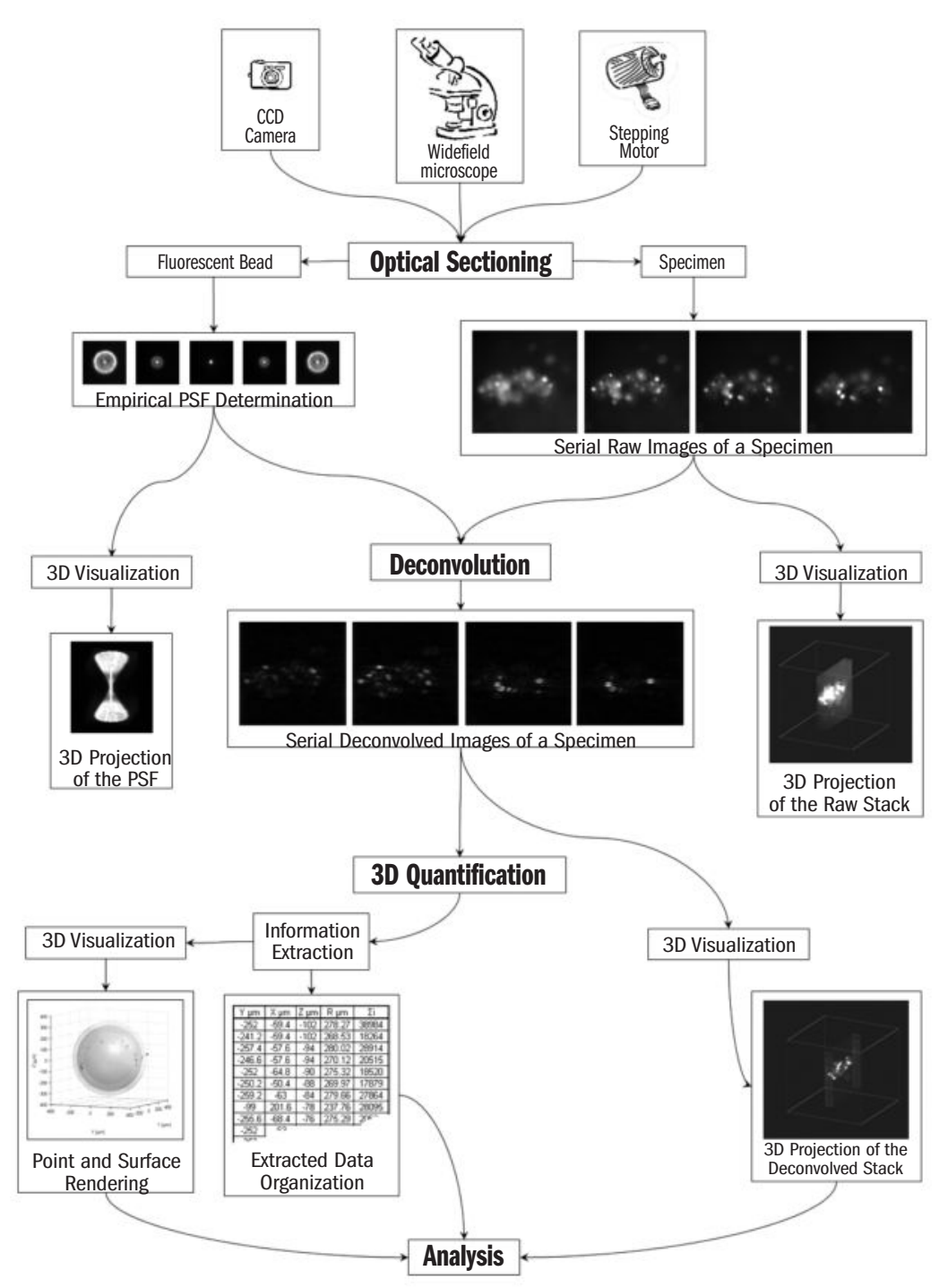


the latter, we used hepatocytes apoptosis in spheroids employing a TUNEL assay method.

Hepatocytes cultured as spheroids are the key functional element of bio-artificial livers (BALs), which are a kind of device in which patient plasma is circulated extracorporeally through a bioreactor that houses metabolically active liver cells sandwiched between artificial plates or capillaries. BALs implementation is one of the most important objectives of many research groups. The main therapeutic use is to treat patients with acute liver failure and patients waiting for liver transplantation (Strain, 2002; Sakai, 1999). The aggregate culture of rat or porcine hepatocytes has been demonstrated to be superior to conventional culture techniques (Klieser, 1997; Griffit, 2002). Therefore, various research groups have attempted to use this approach in bio-artificial liver systems (Sakai, 1999; Lorenti, 2003). However, hepatocyte longterm viability and function maintenance in actual operating bio-artificial liver systems is still difficult to obtain (Sakai, 2001). Hence, how cell viability in spheroids improves using this new technique must be evaluated. For these reasons, appropriate and precise tools for 3D quantification are critical.

Hepatocytes in spheroids retain many morphological characteristics of those in vivo, including the polyhedral shape. Shape conservation is intimately related to the organization of the cytoskeleton, which in spheroids resembles that found in vivo. This kind of culture establishes more cell-cell contacts than other types such as monolayers, and maintains many differentiated functions (Hamilton, 2001). Therefore, spheroids are three-dimensional structures with well-defined morphological features, which determine the proper physiological functionality. Any change in the 3D morphology of the spheroids may produce distortions in their ability to maintain differentiated hepatic-specific cellular functions.

To evaluate hepatocyte formation in spheroids, 2D techniques - such as electron microscopy, phase contrast microscopy and immunohistochemistry are usually used (Klieser, 1997; Hamilton, 2001; Jiang, 2002; Lorenti, 2006). In order to improve this kind of analyses, we show how a modern 3D microscopy technique combined with automatic quantification contributes with very significant results. To achieve this, cell necrosis and apoptosis in spheroids growing in culture were visualized, using the TdT-mediated dUTP nick end labeling
(TUNEL) technique. Additionally, for the first time, apoptotic bodies were automatically quantified in relation to the three different volume zones, considering the number of fragmented-DNA cells, apoptotic bodies, inside the spheroid.

\section{Materials and Methods}

\section{Microscopy system}

The system used to obtain the optical sections of spheroids was developed in our laboratory and it is shown in Figure 2. It is based on an Olympus BX50 upright microscope equipped with both an UV lamp for epi-fluorescence and a halogen lamp for transmitted light. The recording system consists of an Apogee CCD camera Model AM4, attached to the microscope, with a sensor of $768 \times 512$ pixel $^{2}$, square pixels with sides 9 micrometers long, 14 bits of resolution and cooling system. In addition, a stepping motor RS 440-436 was connected to the micrometric screw of the microscope, via a 1:100

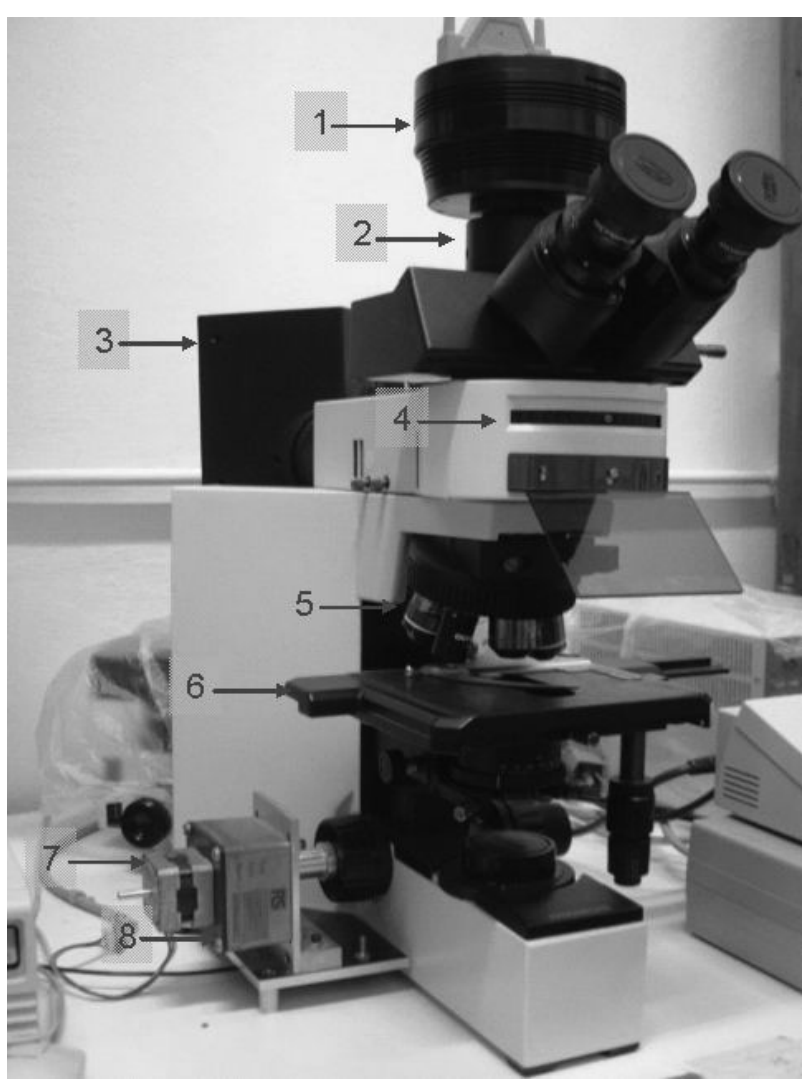

Figure 2. Microscope. (1) CCD camera. (2) C-Mount optical attachment. (3) UV-Lamp placed for episcopic illumination. (4) Dichroic mirror selector. (5) Objective lens. (6) Stage. (7) Stepping motor. (8) Reduction box 1:100. 
reducing box (Adur and Schlegel, 1997). We have also developed a software named SUMDD (DiazZamboni, 2004), which controls both the camera via an ISA card and the stepping motor via parallel port, allowing image capture, focusing by keyboard, optical sectioning, 3D deconvolution and visualization.

\section{Point spread function determination}

Fluorescent beads of 2 micrometers ( $\mu \mathrm{m})$ were optically sectioned with a 10x objective UPIanApo lens, to obtain the corresponding PSF. They were selected to be close to the resolution limit of the microscope and optically sectioned from bottom to top following modified Kozubek's protocols (Kozubek, 2001). Each image was an average of three captures, which were then background corrected. Images were finally stored in TIFF format, gray scale with 8 bits of resolution.

\section{Optical sectioning and deconvolution algorithm}

An optical-section series of $2 \mu \mathrm{m}$ of thickness was obtained using the 10x objective, for each spheroid. The number of sections was selected, guaranteeing that the set of optical sections would contain the whole specimen. The conditions of image capture were the same to those used for the PSF determination. The $X Y$ resolution was selected to be $512 \times 512$ pixel $^{2}$, allowing the largest possible image for fast processing. Our deconvolution algorithms (Diaz-Zamboni, 2003a) use an implementation of the Fast Fourier Transform (FFT), which requires that the total amount of elements is a power of 2 .

Total magnification of the system was determined multiplying the magnification of the objective by the magnification of the lens coupled to the camera $(0.5 x)$. Then, we made use of the magnification formula, which establishes that the square of the total magnification of an optical set up is the square root of the division of the image plane area by the object plane area, as is shown in Equation 1.

$$
\text { Total magnification }=\sqrt{\frac{\text { CCD Sensor Size }}{\text { Field of View }}} \text { Eq. } 1
$$

Since the total magnification, number of pixels in $X$ and $Y$ directions, and CCD sensor pixel size are known, it was possible to calculate the pixel size in the object plane, which resulted in $1.8 \times 1.8 \mu \mathrm{m}^{2}$.

The camera was cooled down to between $-11^{\circ} \mathrm{C}$ and $-15^{\circ} \mathrm{C}$, in order to reduce electronic noise in the image acquisition process, consequently increasing the signal-noise ratio. Images captured were background corrected and stored in TIFF format, gray scale with 8 bits of resolution.

At the same time, a series of transmitted light images were obtained in the same focal planes to have a reference of the spheroid contour.

Spheroids were mounted on slices, which were marked with a thin line on one side, to have a reference of the bottom of the spheroids. In this way, the line was manually focused, constituting the starting point of the sectioning process. Each spheroid was then localized near the line without moving the stage in the axial direction until it fit inside the field of view and the optical sectioning process started, moving the stage downwards automatically, so images in different focal planes were taken from bottom to top of the spheroid.

Digital deconvolution was done after the optical sectioning process, in order to correct the optical system's distortions using the PSF. This was done with an implementation (Diaz-Zamboni, 2003b) of a regularized least square algorithm (Preza, 1992) to deconvolve 3D data sets.

Several types of deconvolution methods exist, which are classified by the convolution model implemented. A good approach to the formalism of these algorithms is given in recent publications (Sarder, 2006) (Sibarita, 2005). However, it is convenient to divide deconvolution algorithms in two groups: deblurring and restoration. The former include those algorithms that are fundamentally $2 \mathrm{D}$, since they apply a plane-by-plane operation to each 2D section of a 3D image stack. The latter are accurately 3D because they operate simultaneously on every voxel in a 3D image stack (Wallace, 2001).

Deblurring algorithms are characterized for removing or eliminating out-of-focus information; they are easy to compute and operate very fast. These algorithms are useful as a first attempt to visualize object structure. However, they are not recommended in quantification procedures because image intensities are removing in an unknown proportion. On the other hand, restoration methods reassign defocused information to its original position in the 3D image stack. Commonly, some implementations of restoration algorithms are slower than deblurring methods because the majority are iterative, which implies a repetitive computational task with the goal of finding a solution that approx- 
imates the real specimen. Nevertheless, one-step restoration algorithms are faster than iterative ones and similar to deblurring methods. The regularized least square method is a precise and fast restoration method useful when quantification is involved (Preza, 1992).

\section{Spheroids}

From a set of porcine hepatic spheroids, three were selected by diameter and fixed with $4 \%$ formaldehyde in $0.1 \mathrm{M} \mathrm{PBS} \mathrm{pH} 7.2$ and stored at $4^{\circ} \mathrm{C}$. The spheroid's mean diameter was obtained by averaging the longest and shortest diameters both using a caliper and by digital image measurement. The spheroids selected had $524 \mu \mathrm{m}, 622 \mu \mathrm{m}$, and $682 \mu \mathrm{m}$ of mean diameter.

The spheroids were washed with PBS $1 X$, permeated with Triton $0.1 \%$ X-100 and $0.1 \%$ Sodium Citrate for 25 minutes, then washed again twice with PBS IX. TUNEL reactions were carried out according to the supplier's instructions (Boehringer Mannheim $\mathrm{GmbH}$. In situ cell death detection fluorescein kit). Next, the spheroids were washed three times more with PBS $3 X$, and possible aqueous remnants were eliminated. Finally, spheroids were mounted with Vectashield (Vector) and optically sectioned with a $10 \mathrm{x}$ objective lens using a $2 \mu \mathrm{m}$ zstepping.

\section{D quantification algorithm}

A special algorithm, based on the background of the model-based methods, was developed to quantify apoptosis in spheroids. In these methods, the model represents the shape and local gray level structure of a target object in an image (Cootes, 2000). The proposed algorithm implements a model that is applied to deconvolved images of apoptotic bodies in spheroids, whose signal is obtained by TUNEL.

The first assumption made was that each spheroid was spherical. The mean radius was obtained by digital image measurement. In order to achieve this, a thresholding process was used, which is a segmentation procedure useful for information extraction (Castleman, 1996). Since the hepatic spheroid is not transparent to visible light, its transmitted light microscopy image is highly contrasted. Image analysis showed a bimodal histogram, therefore its valley's intensity range was used to determine spheroid contour. Next, images were binarized so that all values that were between those threshold levels were converted to 255 and the others to zero. For radius measurements we selected the central image of the stack because it has information of the spheroid equatorial circumference. The radius of each spheroid was obtained tracing lines diametrically along the minor and major diameters of the spheroid, measuring their length, averaging them and then dividing by two. This value was called R. This confirmed the manual selection of spheroids previously done using the caliper. This process was also done in other three images equally spaced in the stack and then logically added to corresponding images of the deconvolved stack to have a reference for $3 \mathrm{D}$ visualization.

In order to determine the distribution of the apoptotic bodies in a spheroid relative to its center, the centroid was established tracing two lines diametrically, corresponding to the minor and major diameters of the spheroid. These coordinates were called $X_{c}, Y_{c}$. A third coordinate $Z_{c}$ was previously determined; since optical sectioning was done guaranteeing that the spheroid equator was in the middle of the stack. Hence, the distance of any point with cartesian coordinate $(x, y, z)$ can be written relative to the centroid of the spheroid in the following way:

$$
r_{p}=\sqrt{\left(x-X_{c}\right)^{2}+\left(y-Y_{c}\right)^{2}+\left(z-Z_{c}\right)^{2}} \quad \text { Eq. } 2
$$

Those points with $r_{p}$ between zero and $R$ ( $0 \leq$ $r_{p}<R$ ) were considered to be inside the spheroid; a criterion implemented considered which points were inside and which were outside the spheroid.

A small bright sphere was used to model an apoptotic body. It can be seen in the deconvolved stack as a brilliant small spherical body with an even brighter center. For our purposes the minimum radius allowed was $2 \mu \mathrm{m}$ and the maximum was 10 $\mu \mathrm{m}$, because this is the size range of a normal hepatocyte nucleus (Hamilton, 2001). These values were called the minimum $\left(r_{\text {min.ap }}\right)$ and maximum $\left(r_{\text {max.ap }}\right)$ apoptotic radius respectively.

Fluorescence retrieved with an acquisition device has a range which is a set of consecutive values usually given in gray levels. In general, fluorescence signals of biological interest are in the superior segment of the dynamic range available of the acquisition device. Particularly, when a signal is captured with a CCD camera, its dynamic range lies between 0 and $2^{n}-1$; where $n$ is the resolution of the camera in bits, and it determines all possible gray levels 
of a fluorescence digital image (Swedlow, 1997). Thus, more formally, the fluorescence signal ranges between a minimum and $2^{n}-1$. These values are referred to as minimum fluorescent signal $(m)$ and maximum fluorescent signal $(M)$. Consequently, any value between 0 and $\mathrm{m}$ is considered as background and values between $m$ and $M$ are biological fluorescent signal.

Since each apoptotic body was approximated by a small brilliant sphere, it was necessary to define parameters that consider both geometry and intensity. To solve this, minimum and maximum integrated intensity values were defined. Minimum integrated intensity (mii) is defined as the sum of voxels intensities -the three-dimensional analogue of a pixel- of the smallest and dimmest sphere that represents an apoptotic body. That is, the sphere with radius $r_{\text {min.ap }}$ in which all voxels have the minimum intensity level considered as fluorescent signal, m. Equation 3 resumes the calculus of the mentioned parameter.

$$
m_{i i}=m\left[\frac{4 \pi r^{3} \text { min.ap }}{3}\right]
$$

In the same way, maximum integrated intensity ( $M_{i i}$ ) was determined using $M$ and $r_{\text {max.ap }}$ as shown in Equation 4.

$$
M_{i i}=\mathrm{M}\left[\frac{4 \pi r^{3} \text { max.ap }}{3}\right]
$$

Equations 3 and 4 result from the solution of the analytical integral of the intensity functions in the spherical volume, defined by $r_{\text {min.ap }}$ and $r_{\text {max.ap }}$ respectively. Since intensity in both cases is a constant $-m$ and $M$, respectively-, the integral's outcome is the multiplication of the constant by the volume of the sphere, which corresponds to the second factor of the equations, in between brackets. Figure 3 shows a depiction of the apoptotic body model previously explained; intensity line profiles are also presented to illustrate the parameters proposed. This must be extended to 3D in order to be in accordance with the mathematical analysis presented.

Finally, automatic counting of apoptotic bodies was implemented in calculus software. That is to say, a computer program loads a complete optically-sectioned stack and decides which apoptotic bodies are inside of a spheroid and which are not, depending on the values of the parameters previously defined. Then, it analyzes if there is any point in the volume (voxel) which falls in the intensity range considered as fluorescent signal, between $m$ and $M$. If there is no point in that range, the algorithm stops. Otherwise, the algorithm examines the integrated intensity around them. An apoptotic element is recognized as such if it has an integrated intensity value between $m_{i i}$ and $M_{i i}$. This is evaluated summing the intensity of the voxels up to $r_{\text {min.ap }}$ and then up to $r_{\text {max.ap. }}$ Finally, the zone processed is darkened with zeros, and the algorithm will not recognize it again in the following iteration. Figure 4 shows a flow diagram of the algorithm.

The output of the algorithm consists on a list of the apoptotic bodies detected. This also contains their centers in cartesian and radial coordinates, relative to the spheroid centroid and its integrated intensity.

For 3D visual analysis, the coordinates of apoptotic bodies' centers were used to render points in
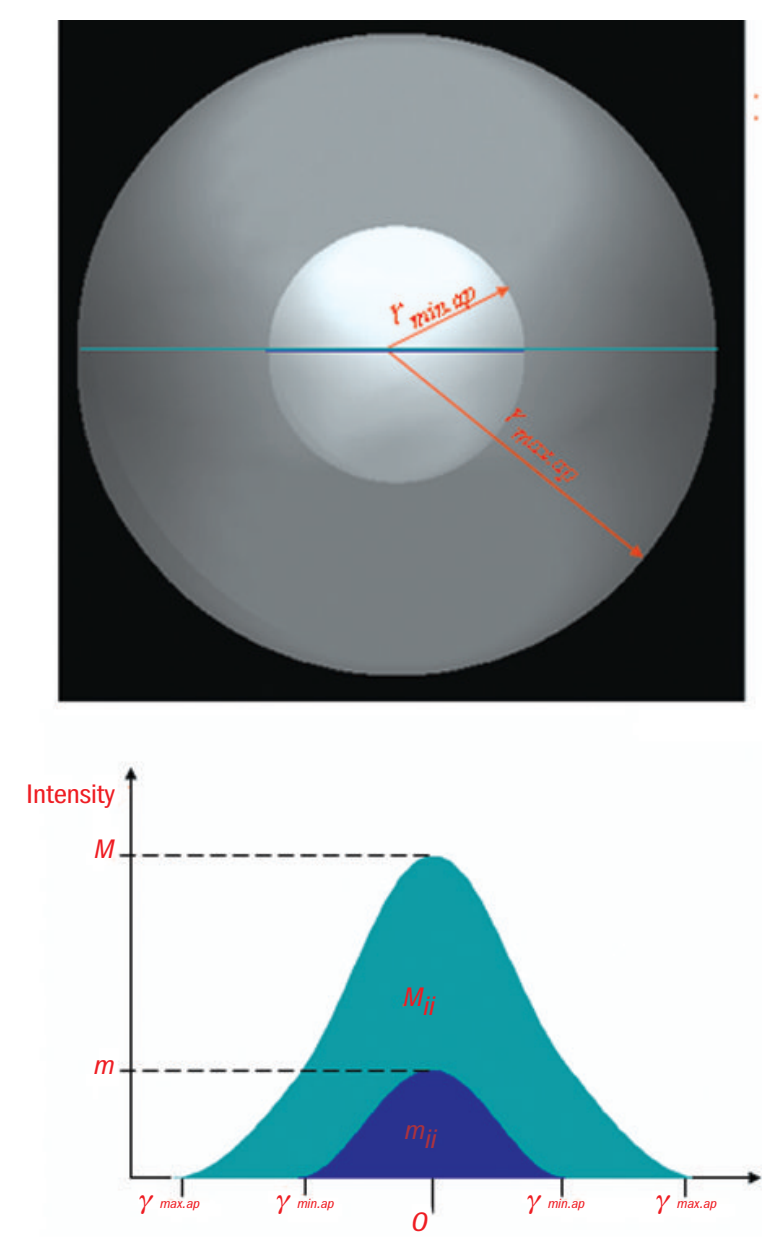

Figure 3. Apoptotic body scheme. Top: largest and smallest expected apoptotic bodies. Low: relationship between intensity line profiles and associated parameters determined for one dimension. 
an environment of 3D reconstruction. These conditions allowed us to perform analyses from different angles fast enough to enable a good 3D animation.

Table 1 resumes the input parameters used to analyze each spheroid.

\section{Analysis of apoptotic body distribution}

Each spheroid was divided into three volumes of the same size. An inner zone named Zone 1, a middle zone called Zone 2 and an outer shell zone named Zone 3. That is to say, volumes $V_{1}, V_{2}$ and $V_{3}$, respectively, where $V_{1}=V_{2}=V_{3}$, which can be written as shown in Equation 5.

$$
\frac{4 \pi r_{1}^{3}}{3}=\frac{4 \pi\left(r_{2}^{3}-r_{1}^{3}\right)}{3}=\frac{4 \pi\left(R^{3}-r_{2}^{3}\right)}{3}
$$

Where, $r_{1}$ defines Zone $1 ; r_{2}-r_{1}$, Zone 2; and $R-r_{2}$, Zone 3. Then, when these equalities are solved to find $r_{1}$ and $r_{2}$, it is possible to write them in terms of $R$. In other words, $r_{1}=69.34 \%$ of $R$ and $r_{2}=87.36 \%$ of $R$. The algorithm's output was corrected consid-

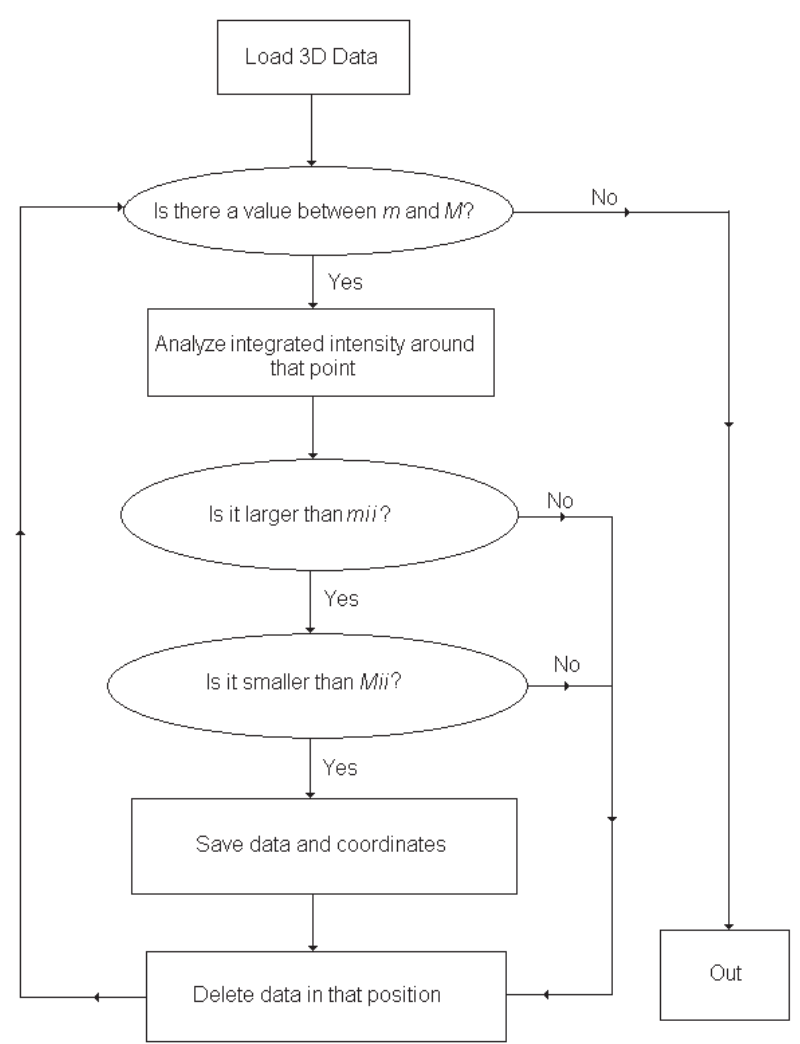

Figure 4. Flow diagram of the logic of the quantification algorithm used to count apoptotic bodies in porcine-hepatocyte spheroids. ering the mean radius of spheroids and it was statistically treated to determine the distribution of apoptotic bodies in the volume. In order to do so, radial coordinates of each apoptotic body detected was normalized relative to the mean radius of spheroids.

\section{Results}

In order to obtain good results in deconvolution it is important to capture the specimen and the PSF in the same conditions, which must be optimal for the microscope system to obtain the best restoration. To achieve this, the captured PSF should be symmetric, that is to say, independent of optical constructive aberrations; the set up should be optimally tuned so as to obtain as symmetric a PSF as possible (Kozubek, 2001). This is done by analyzing different PSFs in dissimilar optical conditions, taking into account axial $(Z)$ and radial $(X-Y)$ symmetry. In general, objective lenses are corrected for spherical aberrations therefore it is common to find very good radial symmetry. However, axial symmetry is highly dependent on the refractive index of the different mediums involved, so it is very important to select correct optical elements (slide glasses and coverslips), immersion oils and mounting mediums (Adur, 2006). As an example of axial asymmetry, Figure 5 shows three different PSFs which correspond to three different coverslip thicknesses. Left and right images show an aggravated asymmetry which is not observed in the middle figure.

Figures $6 \mathrm{a}$ and $6 \mathrm{~b}$ show maximum intensity projections of the $3 \mathrm{D}$ reconstruction of optical sections before and after deconvolution, respectively. Conventional fluorescent volumes contain in-focus information together with out-of-focus contributions from the remainder of the specimen. Thus, on

Table 1. Input parameters used for the correct execution of the algorithm and their respective values used for each spheroid.

\begin{tabular}{lllll}
\hline Parameter & Meaning & \multicolumn{3}{c}{ Value used } \\
& & Sph1 & Sph2 & Sph3 \\
\hline $\mathrm{R}$ & Mean radius of the spheroid & $304.25 \mu \mathrm{m}$ & $249.5 \mu \mathrm{m}$ & $352 \mu \mathrm{m}$ \\
$\mathrm{m}$ & Minimum fluorescent signal & 20 & 20 & 20 \\
$\mathrm{M}$ & Maximum fluorescent signal & 255 & 255 & 255 \\
$\mathrm{r}_{\text {min.ap }}$ & Maximum apoptotic radius & $2 \mu \mathrm{m}$ & $2 \mu \mathrm{m}$ & $2 \mu \mathrm{m}$ \\
$\mathrm{r}_{\text {max.ap }}$ & Minimum apoptotic radius & $10 \mu \mathrm{m}$ & $10 \mu \mathrm{m}$ & $10 \mu \mathrm{m}$ \\
\hline
\end{tabular}


a 3D raw image it is difficult to identify and quantify apoptotic bodies (Figure 6a). However, after restoring the out-of-focus information to its corresponding spatial position, using a specific deconvolution algorithm (Preza, 1992; Diaz-Zamboni, 2003b), it is possible to identify the biological signal. In the present work, in addition to having a visual reference of the position of a specific signal, we logically add the perimeter of the spheroid in some optical sections, which were obtained by thresholding their analogous transmitted microscopy images (Figure 6b).

For better analysis, a second projection is shown in Figure 7, which corresponds to a portion of the volume projected in Figure 6. In this projection, contrast and axial resolution (parallel to red lines) of the deconvolved image (right) are improved. Details in deconvolved projection are noticeable whereas in raw projection they are obscure by defocus information.

Visual analysis of apoptotic body distribution was done using the output data set of the quantification algorithm. Some projective planes are shown in Figures $8 \mathrm{a}, 8 \mathrm{~b}$ and $8 \mathrm{c}$. Apoptotic bodies are represented by points rendered in the coordinates of apoptotic bodies' centers. This improves performance since the complete volume is not rendered, but only important information is. With the purpose of enhancing analysis, delimitating surfaces (which represent boundaries between Zones 1,2 and 3) are also rendered in these figures. By viewing these projections, the density of apoptotic body distribution inside the spheroid is noted. Nevertheless, in order to quantify, a more rigorous analysis is necessary.

Statistics treatment of the algorithm's output was performed to complete the 3D quantification analysis. In order to do this, apoptotic bodies were classified according to their position. Then, the mean and standard deviation of the relative posi-

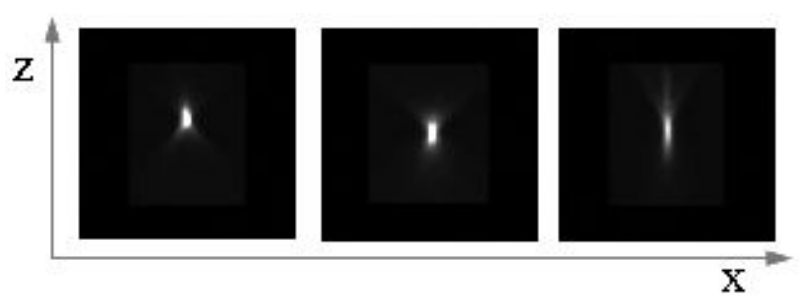

Figure 5. Point spread function selection. Left and right images show PSFs obtained with non-optimal optical paths (coverslips thinner and thicker than $\mathbf{1 . 7} \mu \mathrm{m}$, respectively), while the central picture presents an axially symmetric PSF (coverslip of $\mathbf{1 . 7}$ $\mu \mathrm{m}$ of thickness). a

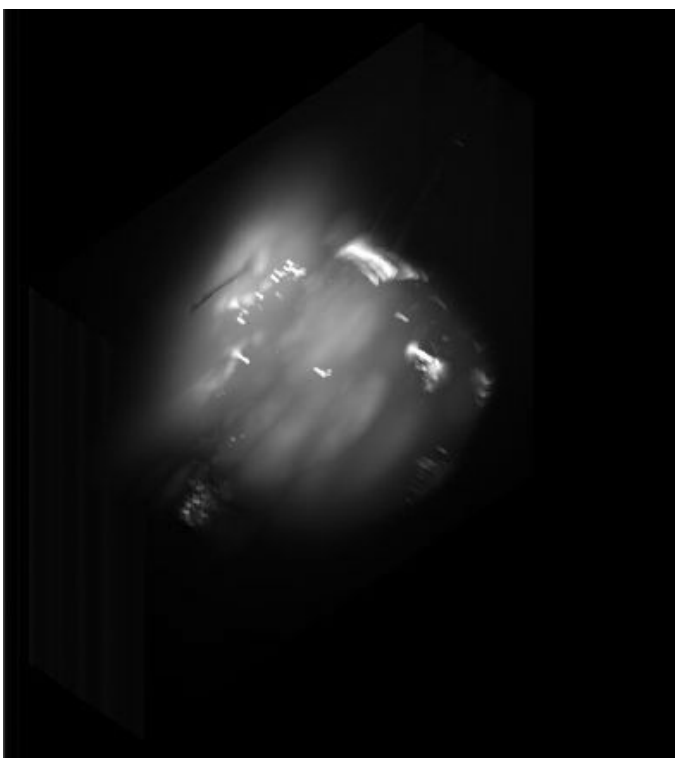

b

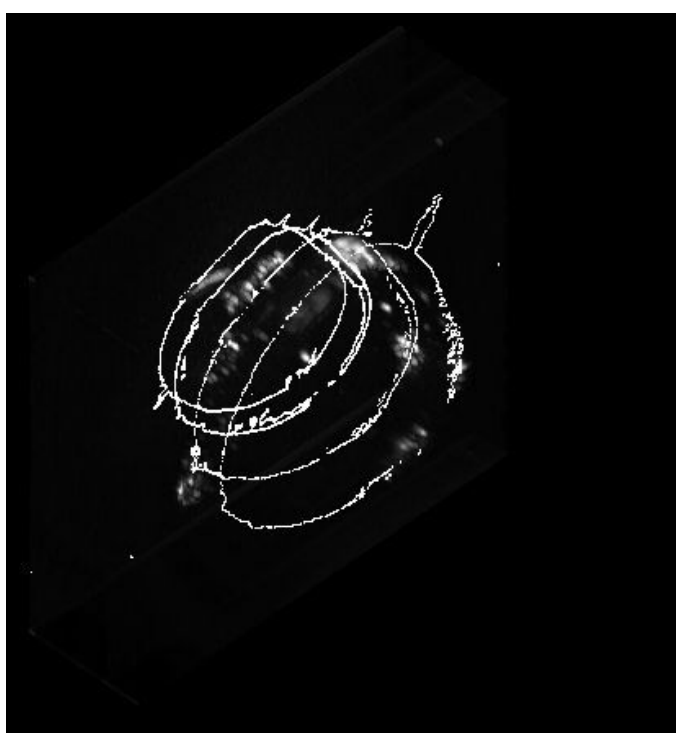

Figure 6. Maximum intensity projections of the 3D reconstruction of optical sections. (a) Projection of the raw volume. (b) Projection of the volume after deconvolution and logical addition of spheroid contours.

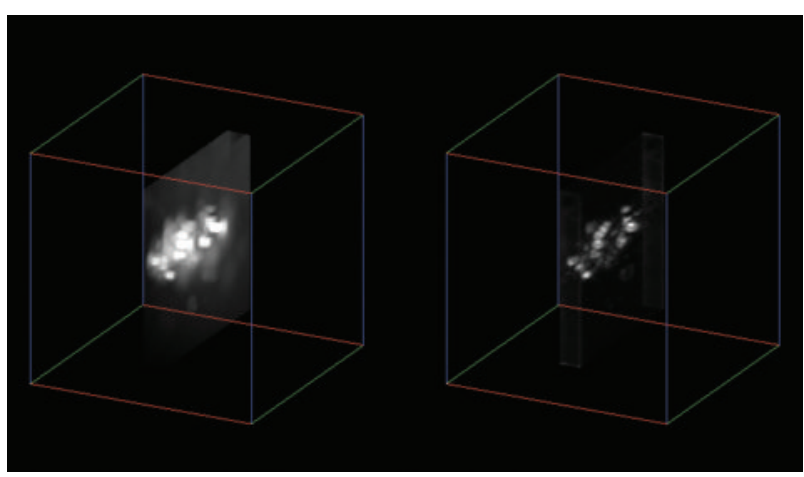

Figure 7. Maximum intensity projection of a portion of a spheroid. The selected subvolume shows that contrast and axial resolution (parallel to red lines) have been improved. 

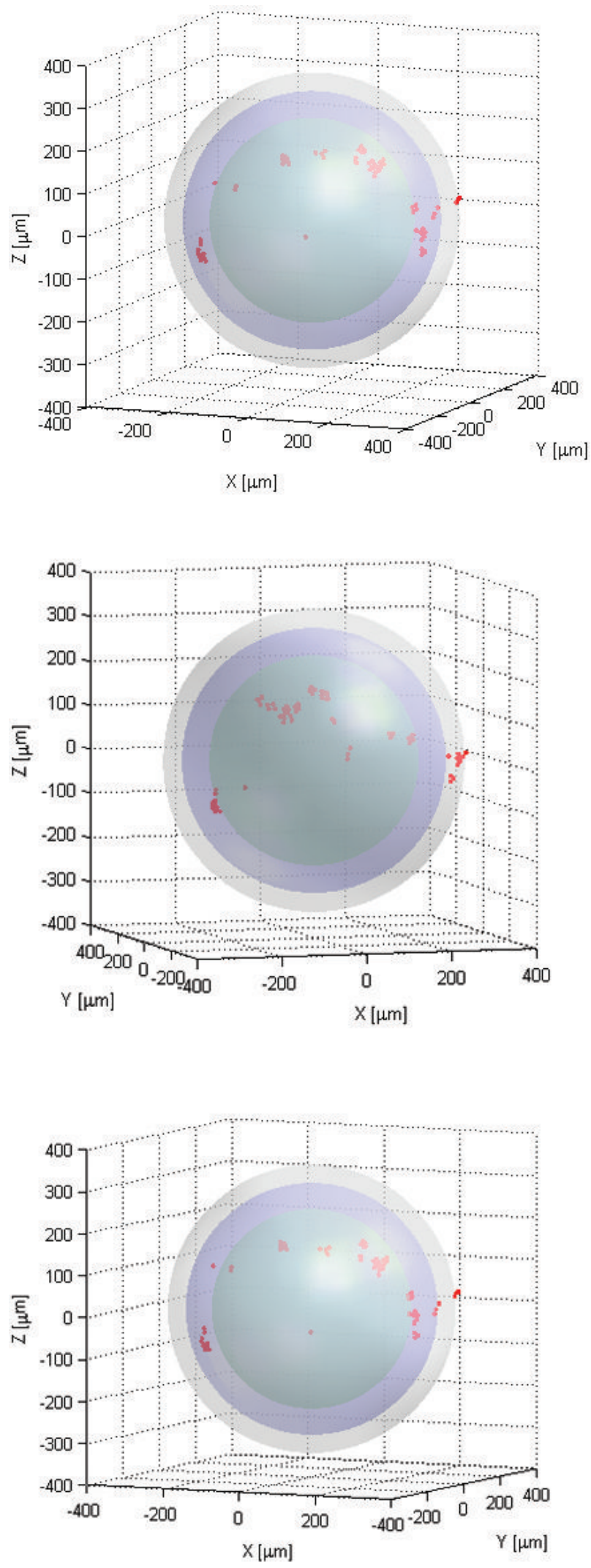

Figure 8. Three different projections of the information extracted with the quantification algorithm allow easy visual analysis. Zones are delimited by spherical surfaces.
Table 2. Number of apoptotic bodies automatically quantified by the algorithm in each one of the three defined zones.

\begin{tabular}{lllll}
\hline Spheroid & 1 & 2 & 3 & Mean $\pm S D$ \\
\hline Zone & Number of apoptotic bodies by mean spheroid radius & \\
\hline 1 & 13.70 & 16.00 & 17.80 & $15.83 \pm 2.05$ \\
2 & 13.66 & 16.20 & 17.78 & $15.88 \pm 2.08$ \\
3 & 04.82 & 05.72 & 06.27 & $05.60 \pm 0.73$ \\
\hline
\end{tabular}

tions were determined for the set of samples.

Most apoptotic bodies were found in Zones 1 and 2. The amount of apoptotic bodies in each region were normalized according to the mean spheroid radius as shown in Table 2.

\section{Discussion}

Confocal and deconvolution microscopy techniques usually provide clean images, but they do not permit the visualization of the complete morphological structure of a specimen at a time. Information extraction processes were implemented to solve this limitation, using a simple model-based method to analyze 3D microscopy images (Cootes, 2000). The border line of spheroids was determined and used to analyze the distribution of apoptotic bodies visually. This 3D tool permits many different views and animations of the specimen; it enables the manipulation of many data sets and automatic processing of them. This also lets us avoid the most tedious work and subjective error source involved in quantification analyses: counting.

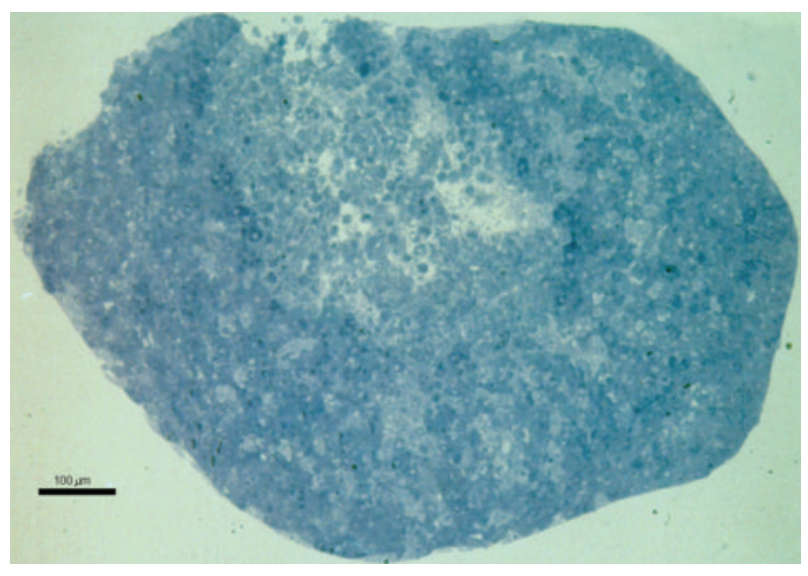

Figure 9. Classical toluidin blue stain protocol shows the lost tissue of a big hepatocyte spheroid. 
Apoptotic body quantification in terms of spheroid size was selected to evaluate the usefulness of this tool. It is known that apoptosis or even necrosis may arise in the central portions of the aggregates when spheroids are grown for extensive periods of time (Lorenti, 2003; Lorenti, 2006). This effect is accentuated as spheroid size increases. In a big spheroid, with radius longer than $300 \mu \mathrm{m}$, apoptosis accumulates in its center and eventually may lead to secondary necrosis; for many years, hypoxia has been proposed as the cause of these (Klieser, 1997).

Using the automatic method of processing presented in this paper, apoptotic bodies were found mainly in the middle region of big spheroids ( 524 $\mu \mathrm{m}, 622 \mu \mathrm{m}$ and $682 \mu \mathrm{m}$ ) but with small difference with to respect to the central region. The small quantity of apoptotic bodies in the central zone could be explained by the effect of material absorption. This phenomenon is observed in Figure 9, where a 2D slice of an equatorial zone was treated with a classical toluidin blue stain protocol.

3D quantification and visualization enable more realistic views and more precise results, and in consequence more objective analysis. There are very small discrepancies with previous $2 \mathrm{D}$ results. New experiments using 3D quantification tools may be performed in the future to extract more information and complement conventional 2D studies.

In conclusion, we presented a computer-based method, which allowed us to show 3D visualization and quantification of apoptotic bodies' fluorescent signal from optical sections of porcine-hepatocyte spheroids, relative to morphological structures, for the first time. This tool is now available to perform 3D analyses on thick specimens or whole embryos, and it is powerful enough to be applied to other kinds of biological phenomena with similar characteristics of intensity and geometry.

As future work, it would be important to undertake a more precise approach to estimate the surfaces making use of a semiautomatic 3D segmentation method that employs gray levels or gradients to extract border information of spheroids, which would be rendered using active contours. The main problem of this kind of reconstruction is that it is highly dependent on the segmentation method; therefore, it is convenient to determine the optimum segmentation procedure for fitting a surface that represents the mean actual boundary of the spheroid.
A final objective should be to estimate the number of apoptotic cells relative to spheroid form and size. To achieve this, fluorochrome concentration should be determined to calibrate the system in order to predict individual apoptotic cell based upon fluorescence intensity of apoptotic bodies; however to achieve this, it is necessary to standardize concentrations versus intensities in a widefield microscope by means of calibrations curves (Fink, 1998).

The methodology presented in this paper is applicable to any 3D microscope. Nevertheless, it is crucial to analyze the 3D information that the system captures in order to adapt the method to other microscopy set-ups such as scanning-confocal or 4pi-confocal microscopy.

\section{Acknowledgements}

The specimens were kindly provided by Dr. Pablo F. Argibay from Instituto de Ciencias Básicas y Medicina Experimental, Hospital Italiano de Buenos Aires, Potosí 4240, C1199ACL, Buenos Aires, Argentina.

\section{References}

Adur JF \& Schlegel JO. Diseño, desarrollo y construcción de un sistema de avance micrométrico para microscopios fotónicos. Degree Thesis. Facultad de Bioingeniería. Universidad Nacional de Entre Ríos, Oro Verde, Entre Ríos, Argentina, 1997.

Adur JF. Determinación de las Propiedades Ópticas de un Sistema de Epifluorescencia y su Utilización en Estudios de Microscopía Cuantitativa 3D. Biomedical Engineer Magister Thesis. Facultad de Ingeniería. Universidad Nacional de Entre Ríos. Oro Verde, Entre Ríos, Argentina 2006.

Agard DA. Optical sectioning microscopy: cellular archiquetecture in three dimensions. Ann Rev Biophys Bioeng, 1984; 13:191-219.

Arndt-Jovin DJ, Robert-Nicoud M, Kaufman SJ, Jovin T M. Fluorescen digital imaging microscopy in cell biology. Science 1985;4723:24756.

Castleman K R. Digital Image Processing. Prentice Hall. New Jersey. 1996.

Cootes T. Model-based method in analysis of biomedical images. In: Image Processing and Analysis. Baldock R and Graham J Eds. Oxford University Press. Chapter 7, 2000, pp. 223-47.

Chen H, Swedlow JR, Grote M, Sedat J W, Agard D A. The collection, processing, display of digital three-dimensional images of biological specimens. In: Handbook of Biological Confocal Microscopy, Pawley J B Ed. Plenum Press, New York, 1995, Chapter 13,197-209.

Diaz-Zamboni JE, Adur JF, Balducci F, Cherniz AS, Izaguirre MF, Casco VH. Algoritmos de Desconvolución Aplicados a Imágenes 3D en Microscopía de Fluorescencia. In: Jornadas de Ingeniería Clínica e Instalaciones Hospitalarias. Paraná, Argentina, $2003 a$.

Diaz-Zamboni JE, Adur JF, Balducci JF, Cherniz AS, Izaguirre M F, Casco VH. Evaluación de Algoritmos de Desconvolución Aplicados a Imágenes 3D en Microscopia de Fluorescencia. In: XIV Congreso Argentino de Bioingeniería y III Jornadas de Ingeniería Clínica. Córdoba, Argentina, 2003b.

Diaz-Zamboni J E. Software para usuarios de microscopios de desconvolución digital. Degree Thesis. Facultad de Ingeniería, Universidad Nacional de Entre Ríos, Oro Verde, Entre Ríos, 2004.

Fink C, Morgan F, Loew LM. Intracellular fluorescent probe concentrations by confocal microscopy. Biophys J 1998;75:1648-58. 
Griffith LG, Naughton G. Tissue engineering-Current challenges and expanding opportunities. Science 2002; 295:1009-14.

Hamilton GA, Westmoreland C, George E. Effects of medium composition on the morphology and function of rat hepatocytes cultured as spheroids en monolayers. In Vitro Cell Dev Biol Animal 2001; 37:656-67.

Jiang J, Kojima N, Kinoshita T, Miyajima A, Yan W, Sakai Y. Cultivation of fetal liver cells in a three dimensional poly $L$ lactic acid scaffold in the presence of oncostatin M. Cell Transplant 2002; 11:403-06.

Klieser W M. Three-dimensional cell cultures: from molecular mechanisms to clinical applications. American Phisiological Society 1997. C1109-C1123.

Kozubek M. Theoretical versus experimental resolution in optical microscopy. Microsc Res Tech 2001; 53:157-66.

Lorenti AS, Barbich $M$, de Santibanes $M$, Ielpi $M$, Vazquez JC, Sorroche P, Argibay P. Ammonium detoxification performed by porcine hepatocyte spheroids in a bioartificial liver for pediatric use: preliminary report. Artif Organs 2003; 27:665-70.

Lorenti AS, Hidalgo AM, Barbich MR, Torres J, Batalle J, Izaguirre MF, Fiorucci MP, Casco VH, Gadano A, Argibay PF. Structural and functional polarity of porcine hepatocyte cultured spheroids. Acta Gastroenterol Latinoam 2006;36:66-75.

Preza C, Miller MI, Thomas LJ, McNally JG. Regularized linear method for reconstruction of three-dimensional microscopic objects from optical sections. J Opt Soc Am A1992;9:219-28.

Sakai Y, Naruse K, Nagashima I, Muto T, Suzuki M. A new bioartificial liver using porcine hepatocyte spheroids in high cell density sus- pension perfusion culture: in vitro performance in synthesized culture medium and in $100 \%$ human plasma. Cell Transplant 1999; 8:531-41.

Sakai Y, Furukawa K, Ushida T, Tateishi T, Suzuki M. In vitro organization of biohybrid rat liver tissue incorporating growth factor and hormone releasing biodegradable polymer microcapsules. Cell Transplant 2001; 10:479-83.

Sarder P, Nehorai A. Deconvolution methods for 3-D fluorescence microscopy images Signal Processing Magazine, IEEE, 2006; 23:32-45.

Sibarita J. Deconvolution microscopy. Adv Biochem Eng Biotechnol, 2005; $95: 201-43$.

Strain A. J, Neuberger James M. Bioartificial liver - State of the art. Science 2002; 295:1005-14.

Streicher J, Weninger W J, Müller G B. External Marker-Based automatic congruencing a new method of $3 \mathrm{D}$ reconstruction from serial sections. Anat Rec 1997; 248:583-602.

Swedlow $J \mathrm{R}$ et al. Deconvolution in Optical Microscopy. In: Deconvolution of Images and Spectra. Jansson P A, Ed. Academic Press, 1997, Chapter 9, pp. 284-309.

Török É, Pollok J M, Ma P X, Kaufmann P M, Dandri M, Petersen J, et al. Optimization of hepatocyte spheroids formation for hepatic tissue engineering on 3D biodegradable polymer within a flow bioreactor prior to implantation. Cells Tissues Organs 2001; 169:34-41.

Wallace W, Schaefer L H, Swedlow J R. A workingperson's guide to deconvolution in light microscopy. BioTechniques 2001;31:107697. 
J.E. Diaz-Zamboni et al. 